\title{
Prevención de adherencias peritoneales postoperatorias mediante uso de antagonista de receptores de neurokinina tipo 1*
}

\author{
Drs. CARLOS GONZÁLEZ-TORRES ${ }^{1}$, ESTRELLA UZCÁTEGUI P. ${ }^{1}$, MELISSE MILANO M.², \\ JOSÉ PLATA-PATIÑO일 , FABIANNE NOBOA B. ${ }^{1}$, INT. NAOAKI SHIOZAWA C. ${ }^{3}$
}

\author{
Servicio de Cirugía General. Hospital Universitario de Los Andes. \\ Laboratorio de Anatomía Patológica. \\ Interno de Sexto año de Medicina. \\ Facultad de Medicina. Universidad de Los Andes. Mérida, Venezuela.
}

\begin{abstract}
Peritoneal adhesions prevention. Neurokinin-1 receptor antagonist

Background: The fibrinolytic activity plays an important role in Peritoneal Adhesions (PA) development. It's well known that de substance P decreased the fibrinolysis by binding the neurokinin-1 receptor, improving the PA formation. Objectives: To evaluate the effectiveness of intraperitoneal treatment with a Neurokinin-1 receptor antagonist (NK-R1A) in peritoneal adhesion prevention in animal model. Materials and Methods: In 40 male wistar rats, PA were induced, and then randomly assigned to 2 groups: A group treated with Aprepitant (NK-R1A), and a control group. The animals were killed at 7 or 14 postoperative day, and the number, severity and histopathology of PA were evaluated. Results: NK-R1A decreased the number ( $40 \%$ less) and severity $(\mathrm{p}=0.001)$ of PA when compare to control group. The NK-R1A group had less PA in manipulated and no manipulated organs during surgery. Besides presented less fibrosis $(p=0.001)$, less inflamation $(p=0.005)$ and less vascular proliferation $(p=0.047)$ than control group. Conclusions: The NK$\mathrm{R} 1 \mathrm{~A}$ is effective as in PA prevention.
\end{abstract}

Key words: Peritoneal adhesions, Neurokinin 1 receptor antagonist, Substance P.

\section{Resumen}

Introducción: La actividad fibrinolítica juega un papel fundamental en el desarrollo de las adherencias peritoneales (AP), y se conoce que la Sustancia $\mathrm{P}$ al actuar sobre receptores de neurokinina tipo 1 a nivel peritoneal, disminuye la fibrinólisis, favoreciendo la formación de las mismas. Objetivos: Evaluar la efectividad del tratamiento intraperitoneal con antagonista de receptores 1 de neurokinina (NK-R1A) en la prevención de AP en modelo animal. Materiales y Métodos: A 40 ratas wistar se les practicó cirugía formadora de AP y fueron distribuidas de forma aleatoria en 2 grupos, un grupo que recibió Aprepitant (NK-R1A), y el otro como grupo control. Los animales fueron sacrificados a los 7 ó 14 días, y se evaluó el número, severidad e histopatología de las AP. Resultados: El NK-R1A disminuyó el número ( $40 \%$ menos) y severidad de las

\footnotetext{
* Recibido el 10 de Marzo de 2010 y aceptado para publicación el 7 de Abril de 2010.

Correspondencia: Dr. Carlos González T.

Av. 16 de Septiembre. 5101. Mérida, Estado Mérida - Venezuela.

E-mail: cggt80@hotmail.com
} 
AP $(p=0,001)$ en relación al grupo control y presentó menos AP en órganos manipulados y no manipulados durante la cirugía. Además presentó menor grado de fibrosis $(p=0,001)$, menor inflamación $(p=0,005)$ y menor proliferación vascular $(\mathrm{p}=0,047)$ que el grupo control. Conclusión: El tratamiento peritoneal con NK-R1A es eficaz en la prevención de la formación de AP.

Palabras clave: Adherencias peritoneales, antagonista de receptor 1 de neurokinina, Sustancia P.

\section{Introducción}

Las adherencias peritoneales (AP) pueden ser definidas como bandas fibrosas anormales entre órganos y/o tejidos en la cavidad abdominal, que normalmente están separados ${ }^{1-3}$. La gran mayoría de estas son adquiridas como resultado de una injuria peritoneal (ya sea incisión, cauterización, sutura u otro tipo de trauma) siendo la causa más común la cirugía ${ }^{4}$. Independientemente de su origen, las AP van a ser resultado de una compleja interacción de procesos inflamatorios intraperitoneales en los que la cascada de coagulación y el proceso de fibrinólisis van a estar íntimamente relacionados ${ }^{4,5}$.

Se estima que del 93 al $100 \%$ de los pacientes que van a cirugía transperitoneal desarrollarán $\mathrm{AP}^{6}$. Uno de los factores quirúrgicos que han demostrado contribuir a la formación de AP es la presencia de cuerpos extraños intraperitoneales, incluyendo mallas, talco de guantes, material de sutura y litos vesiculares residuales, entre otros ${ }^{7}$. Afortunadamente, la gran mayoría de los pacientes con AP no experimentan ningún síntoma clínico, sin embargo, a corto o largo plazo pueden afectar de forma severa la calidad de vida de millones de personas alrededor del mundo, al condicionar diversos problemas como obstrucción intestinal, dificultad técnica en reintervenciones, dolor crónico e infertilidad femenina, entre otros, siendo por lo tanto causa de una morbilidad y mortalidad significativa ${ }^{5-7}$.

Las AP han sido objeto de investigación desde hace muchos años, siendo reportado el primer caso fatal de obstrucción intestinal por AP en $1872^{8}$. En la actualidad, los estudios sugieren que las AP son la causa principal de obstrucción intestinal en el mundo occidental, ocasionando entre el 60 al $70 \%$ de las obstrucciones de intestino delgado ${ }^{9,10}$.

La terapéutica de mayor utilización ha sido la aplicación de métodos de barreras. Estas barreras, que existen en la forma de membranas o gel, actúan separando las superficies peritoneales lesionadas que puedan estar en riesgo de formar AP. Ejercen su efecto de forma local, justo en el sitio donde han sido aplicados y no tienen efecto en áreas distantes de la cavidad peritoneal. Su utilidad es algo limitada en los casos de anastomosis intestinales y procesos infecciosos intraperitoneales ya que se ha evidenciado un aumento en la filtración de líneas anastomóticas y en la formación de abscesos intrabdomina- les ${ }^{11,12}$. A pesar de esto, es el método aplicado con mayor frecuencia en la práctica clínica ${ }^{13,14}$.

Un enfoque alternativo en la prevención de las AP es la utilización de terapias farmacológicas que inhiben o retardan la formación de las AP, sin proveer una barrera física. Estas intervenciones farmacológicas incluyen el uso de antiinflamatorios (esteroideos y no esteroideos), anticoagulantes y fibrinolíticos, inhibidores y moduladores de factores de crecimiento, así como una gran gama de sustancias dirigidas a atenuar la formación de $\mathrm{AP}^{13,14}$.

Estudios previos han identificado la presencia de Sustancia P (SP) en fluido peritoneal así como en tejido proveniente de AP. La SP pertenece a las takicininas, y sus efectos son mediados por la unión a receptores conocidos como Receptores de Neurokinina 1,2 , y 3 , con mayor selectividad por el NK-R ${ }^{15}$. Reed y $\mathrm{col}^{16}$, en 2002 , demostraron que los niveles de ARNm de SP y NK-R1 están aumentados en el tejido de AP, 3 días posteriores a la cirugía. La SP al actuar sobre el NK-R1 aumenta la expresión del factor de crecimiento tumoral $\beta$ (TGF- $\beta$ ), el cual es una potente citoquina que contribuye a la disminución de la capacidad fibrinolítica peritoneal, mediante la disminución de la actividad de tPA, evitando con esto la disolución temprana de las $\mathrm{AP}^{5}$. Dado estos hallazgos, es evidente que la SP y el NK-R1, juegan un papel de suma importancia en la patogénesis de la formación de AP. Basándose en estos estudios, se plantea el objetivo de evaluar la efectividad del tratamiento intraperitoneal con un antagonista de los NK-R1 en la prevención de adherencias peritoneales en un modelo animal, teniendo como referencia un grupo control.

\section{Material y Método}

\section{Diseño del Estudio}

Se realizó un estudio experimental en modelo animal, con diseño aleatorizado de 1 tratamiento y un grupo control.

\section{Unidad Experimental}

Se utilizaron 40 ratas Wistar macho con peso comprendido entre 250 y 300 gr, distribuidas en 2 grupos de estudio de 20 animales cada uno. Todos los animales se mantuvieron bajo condiciones de humedad y temperatura adecuadas, ciclos de 12 
horas de luz y oscuridad, y provistos de alimento y agua ad libitum.

\section{Procedimiento de Inducción de AP, aplicación de tratamiento intraperitoneal y evaluación:}

Los animales fueron anestesiados mediante el uso de Ketamina intramuscular a dosis de $60 \mathrm{mg} / \mathrm{kg}$. Se realizó una cirugía con efectividad comprobada en la formación de $\mathrm{AP}^{17,18}$, mediante abrasión de la serosa, por frotamiento enérgico con gasa hasta la aparición de puntos hemorrágicos (Figura 1), a nivel de 2 áreas de ciego y en 5 segmentos de $1 \mathrm{~cm}$ de longitud en el intestino delgado (Figura 2).

Adicionalmente se realizaron 4 puntos isquémicos en el peritoneo parietal anterior a cada lado de la línea media, mediante el pinzamiento de aprox 5 $\mathrm{mm}$ de peritoneo con una pinza hemostática y pos-

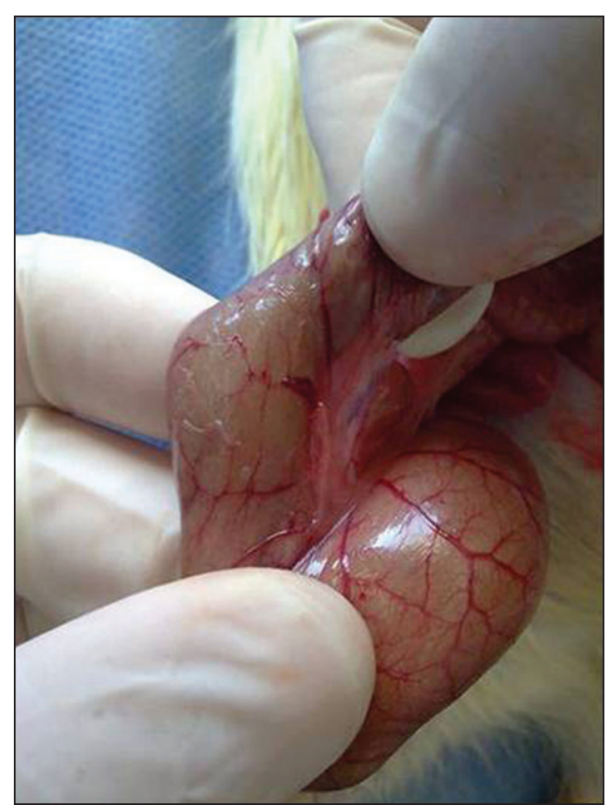

Figura 1. Abrasión de serosa con áreas de hemorragia punteada en intestino delgado. terior ligadura con seda 4-0 (Figura 3).

Luego se asignó de forma aleatoria el animal a cada uno de los grupos de estudio y se procedió a la aplicación del tratamiento intraperitoneal correspondiente:

Grupo C: Grupo control, al cual no se aplicó ninguna terapéutica.

Grupo A: Grupo de estudio, al cual se aplicó un NK-R1A, mediante irrigación en la cavidad peritoneal. Se utilizó el Aprepitant, antagonista altamente selectivo de los NK-R1, droga aprobada para uso clínico por la FDA para el tratamiento de emesis postoperatoria y postquimioterapia. La dosis administrada fue de $25 \mathrm{mg} / \mathrm{kg}$ diluido en $1 \mathrm{ml}$ de solución Dimetilsulfoxido (DMSO) al 100\%.

Durante los 3 primeros días de postoperatorio, los animales se mantuvieron en jaulas individuales

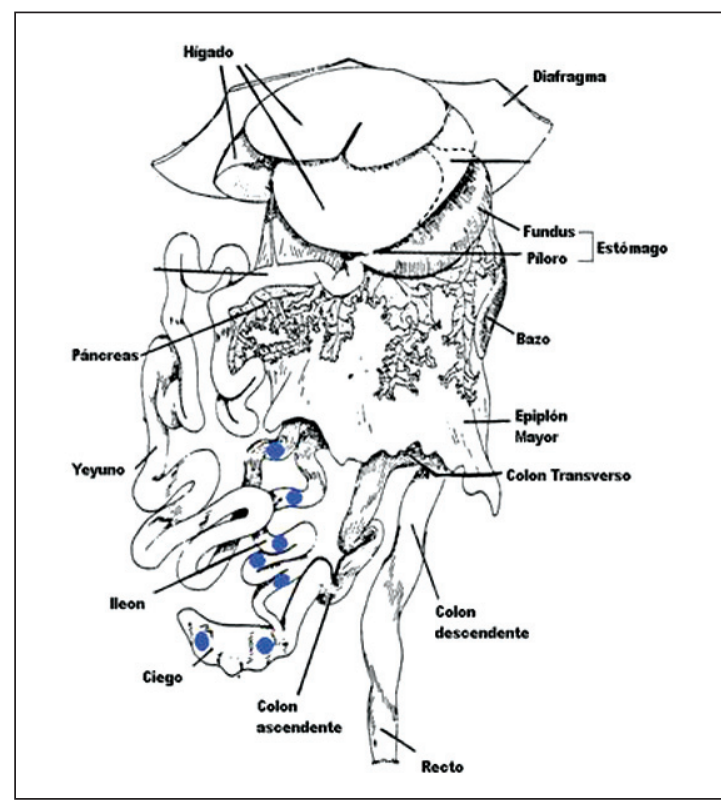

Figura 2. • Áreas de intestino donde se realizó abrasión de serosa.

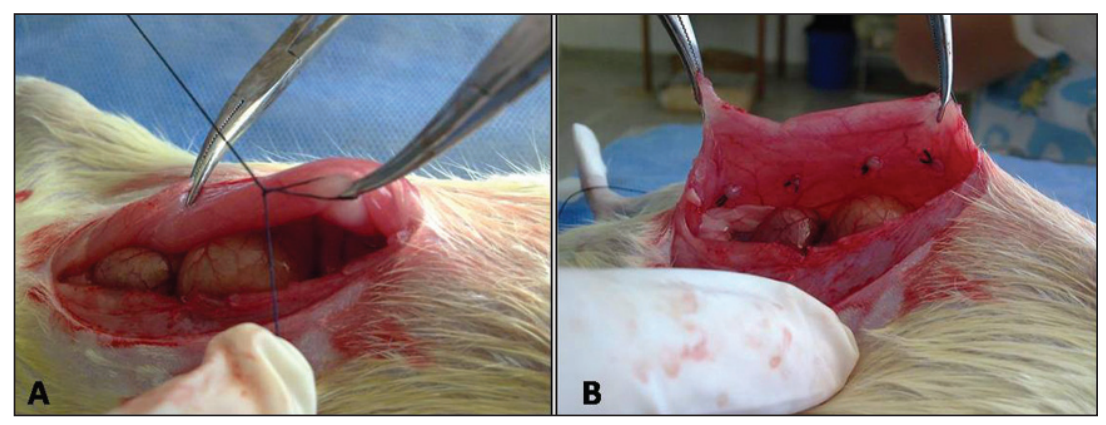

Rev. Chilena de Cirugía. Vol 62 - N 4, Agosto 2010; pág. 369-376
Figura 3. Elaboración de puntos isquémicos en peritoneo parietal anterior.

A: Pinzamiento de peritoneo con pinza hemostática y ligadura con seda 4-0.

$\mathrm{B}$ : Cuatro puntos isquémicos en el peritoneo parietal anterior. 
y recibieron tratamiento antibiótico con Ampicilina/ Sulbactam a dosis de $100 \mathrm{mg} / \mathrm{kg} /$ día, y analgésico con Ketoprofeno a dosis de $5 \mathrm{~m} / \mathrm{kg} /$ día, ambos administrados por vía intramuscular cada 12 horas. Luego fueron agrupados en jaulas comunes de 6 animales.

Los animales fueron sacrificados mediante inhalación de $\mathrm{CO}_{2}$. Se realizó necropsia para evaluar la cavidad peritoneal y determinar la presencia de AP. Se evaluó la severidad de las mismas, mediante la Escala pre-establecida de Mazuji ${ }^{19}$ (Tabla 1) y según el número de puntos isquémicos peritoneales involucrados. Se identificaron las áreas anatómicas involucradas en las AP y se categorizaron en 2 grupos: órganos viscerales manipulados durante la cirugía (Intestino delgado y ciego) y órganos viscerales no manipulados en el transoperatorio. Dicho procedimiento fue realizado por un cirujano que desconocía el grupo al cual pertenecía el animal en estudio.

Además se tomaron muestras de las AP encontradas, para estudio histopatológico. Las mismas fueron fijadas en formaldehido al $10 \%$ e incluidas en parafi- na. Se determinaron los siguientes parámetros histopatológicos: fibrosis (proliferación fibroblástica), inflamación y proliferación vascular. Estos hallazgos se evaluaron cualitativamente y se clasificaron de acuerdo a su intensidad en cuatro grupos (grado 0: negativo, grado 1: leve, grado 2: moderado y grado 3: severo), según la clasificación Kanbour-Shakir et a ${ }^{20}$ modificada (Tabla 2).

\section{Análisis de los Datos}

Las variables cuantitativas fueron expresadas con medidas de tendencia central y dispersión, y las cualitativas fueron expresadas en frecuencias absolutas y/o porcentuales. La comparación entre los grupos se realizó mediante pruebas de Chi-cuadrado de Pearson o Test de Fisher, según el caso, y se consideró que había significancia estadística en las mismas, cuando el valor de $\mathrm{p}$ fue menor de 0,05 . Todos los análisis estadísticos se realizaron utilizando el programa SPSS (Statistical Package for the Social Sciences, version 17.0; SSPS Inc., Chicago, IL).

Tabla 1. Escala de Mazuji para evaluar severidad de las adherencias peritoneales

\begin{tabular}{cl}
\hline Grado & Características de las Adherencias \\
\hline 0 & Sin adherencias \\
1 & Adherencias leves que pueden ser separadas mediante disección roma \\
2 & $\begin{array}{l}\text { Adherencias moderadas en las cuales la mayoría puede ser separada mediante disección roma, pero se requiere } \\
\text { disección cortante en menos del } 50 \% \text { de los segmentos adheridos }\end{array}$ \\
3 & Adherencias severas que requieren disección cortante en más del $50 \%$ de los segmentos adheridos \\
4 & Adherencias severas asociadas a lesiones de serosa \\
5 & Adherencias severas asociadas a lesiones de espesor total de la pared intestinal o parénquima del órgano \\
\hline
\end{tabular}

Fuente: Mazuji et $\mathrm{al}^{19}$.

Tabla 2. Clasificación de parámetros histológicos en adherencias peritoneales

\begin{tabular}{|c|c|c|c|}
\hline & Fibrosis & Inflamación & Proliferación vascular \\
\hline Grado 0 & $\begin{array}{l}\text { Ausencia de proliferación } \\
\text { fibroblástica }\end{array}$ & $\begin{array}{l}\text { Ausencia de componente } \\
\text { inflamatorio }\end{array}$ & $\begin{array}{l}\text { Ausencia de proliferación } \\
\text { vascular }\end{array}$ \\
\hline Grado 1 & Escasa & $\begin{array}{l}\text { Leve: Células gigantes, } \\
\text { ocasionales linfocitos y } \\
\text { plasmocitos }\end{array}$ & Leve \\
\hline Grado 2 & Moderada & $\begin{array}{l}\text { Moderada: Células gigantes, } \\
\text { plasmocitos, eosinófilos y } \\
\text { neutrófilos }\end{array}$ & Moderada \\
\hline Grado 3 & Densa & $\begin{array}{l}\text { Severa: Abundantes células } \\
\text { inflamatorias y microabscesos }\end{array}$ & Severa \\
\hline
\end{tabular}

Fuente: Kanbour-Shakir et al. ${ }^{20}$ modificada por González-Torres C. y Milano M. 


\section{Resultados}

Durante el estudio ocurrieron 8 muertes no planificadas ( $20 \%$ de la muestra total), 5 en el grupo control, y 3 en el grupo NK-R1A, sin diferencia estadística entre los mismos $(\mathrm{p}=0,919)$. Las principales causas de muerte fueron peritonitis secundaria a perforación intestinal desapercibida durante el acto operatorio y obstrucción intestinal secundaria a $\mathrm{AP}$, distribuidas de forma uniforme entre los grupos $(\mathrm{p}=0,980)$.

\section{Evaluación de adherencias peritoneales}

Se evaluó la severidad de las AP viscerales mediante la escala de Mazuji (Figura 4), evidenciándose disminución de la misma en el grupo de tratamiento con $p=0,001$ (Figura 5).

Se determinó el número de puntos isquémicos comprometidos en las AP (figura 6), evidenciándose que los animales que recibieron tratamiento con NK-R1A tuvieron una formación de AP promedio de $20,63 \% \pm 15,85$, mientras que en los del grupo control el promedio de formación de AP fue de $60,63 \pm 26,67$. Esto representa una efectividad en la disminución de la formación de AP del 40\%, siendo la misma estadísticamente significativa, con $\mathrm{p}<$ 0,001 (Figura 6).

Se evidenció que en ambos grupos hubo mayor adherencias en los órganos manipulados durante la
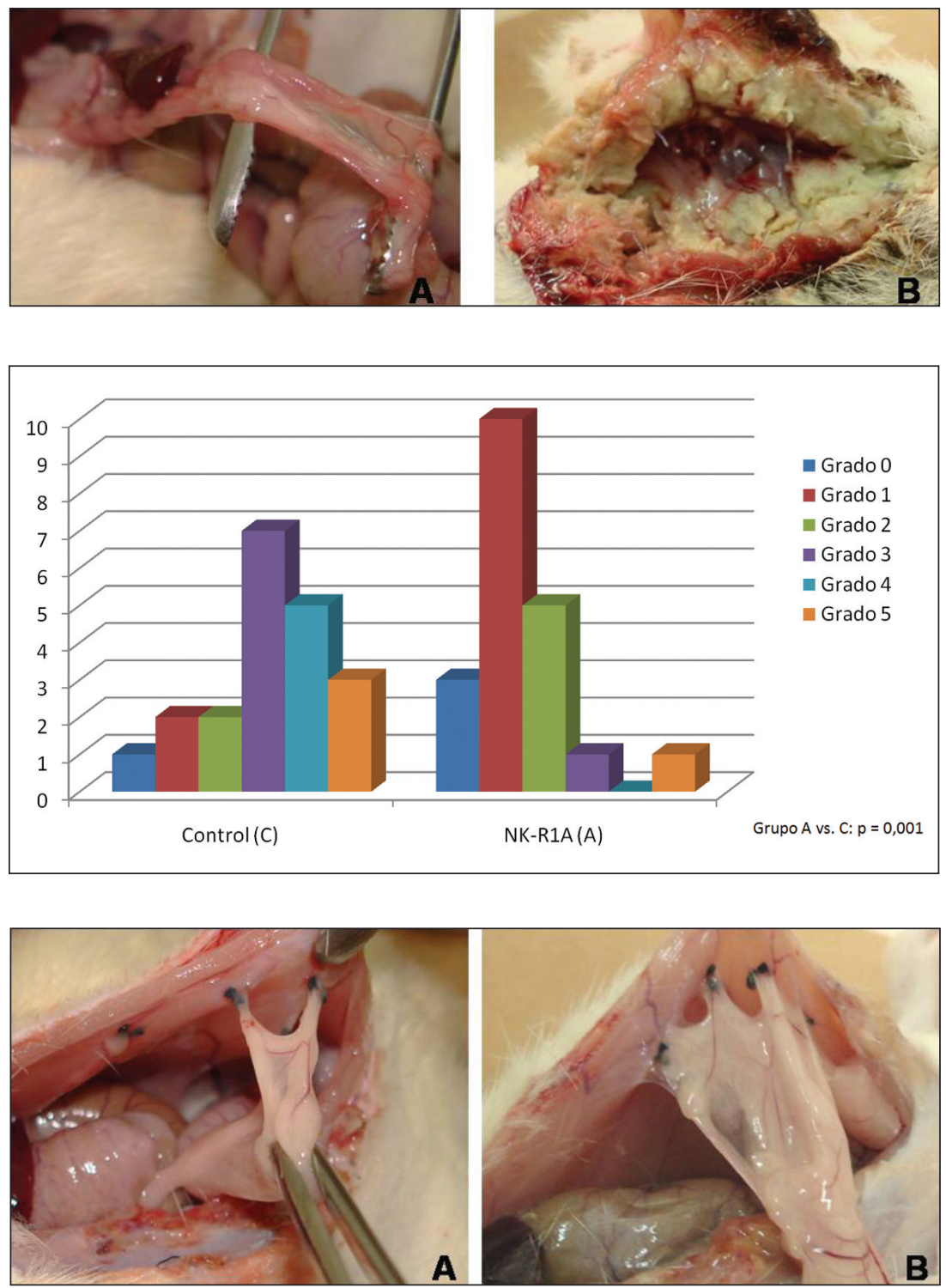

Figura 4. Diferentes grados de severidad de adherencias peritoneales, según escala de Mazuji. A. Adherencias grado 2. B. Adherencias grado 5 .

Figura 5. Severidad de las adherencias peritoneales según escala de Mazuji.

Figura 6. Compromiso de puntos isquémicos en peritoneo parietal anterior. A. Adherencias a 2 puntos parietales $(25 \%)$. B. Gruesas adherencias a 4 puntos isquémicos de la pared $(50 \%)$. 
cirugía ( $p=0,003$, Figura 7), sin embargo, el grupo tratado con NK-R1A presentó de manera significativa menor número de AP tanto en los órganos viscerales manipulados como los no manipulados en el transoperatorio $(\mathrm{p}=0,001$ en ambos casos).

\section{Estudios histológicos}

Los animales tratados con NK-R1A tuvieron menor grado de fibrosis, inflamación y proliferación vascular que el grupo control, siendo esta diferencia estadísticamente significativa (Figuras 8 y 9).
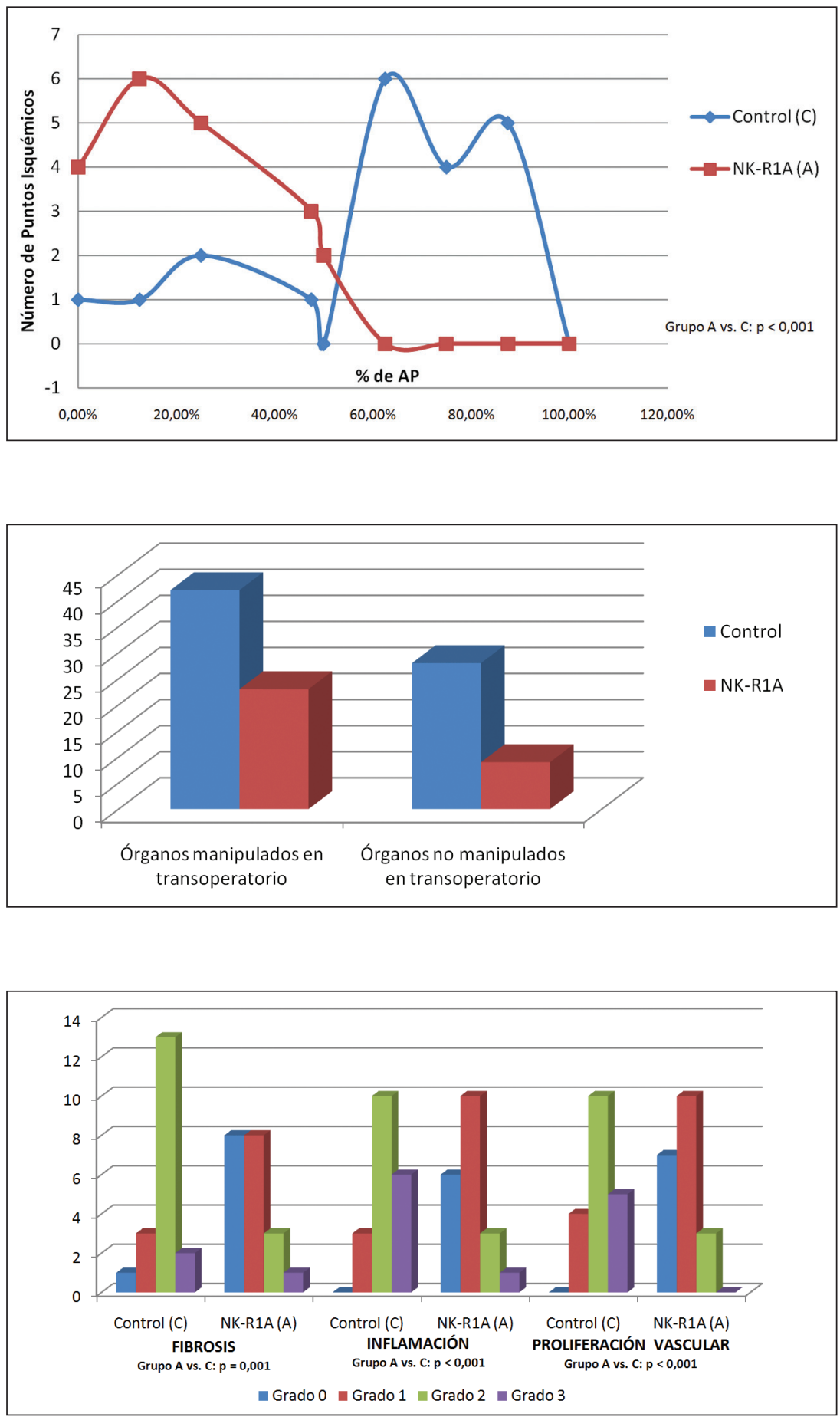

Figura 7. Evaluación cuantitativa de adherencias peritoneales según puntos isquémicos peritoneales. Fuente: Formato de recolección de datos CBBIOULA/008.

Figura 8. Desarrollo de adherencias peritoneales en órganos manipulados o no, durante el acto quirúrgico. Fuente: Formato de recolección de datos $\mathrm{CB}$ BIOULA/008.

Figura 9. Grado de Fibrosis, Inflamación y Proliferación Vascular en adherencias peritoneales postoperatorias. Fuente: Formato de recolección de datos $\mathrm{CB}$ BIOULA/008. 


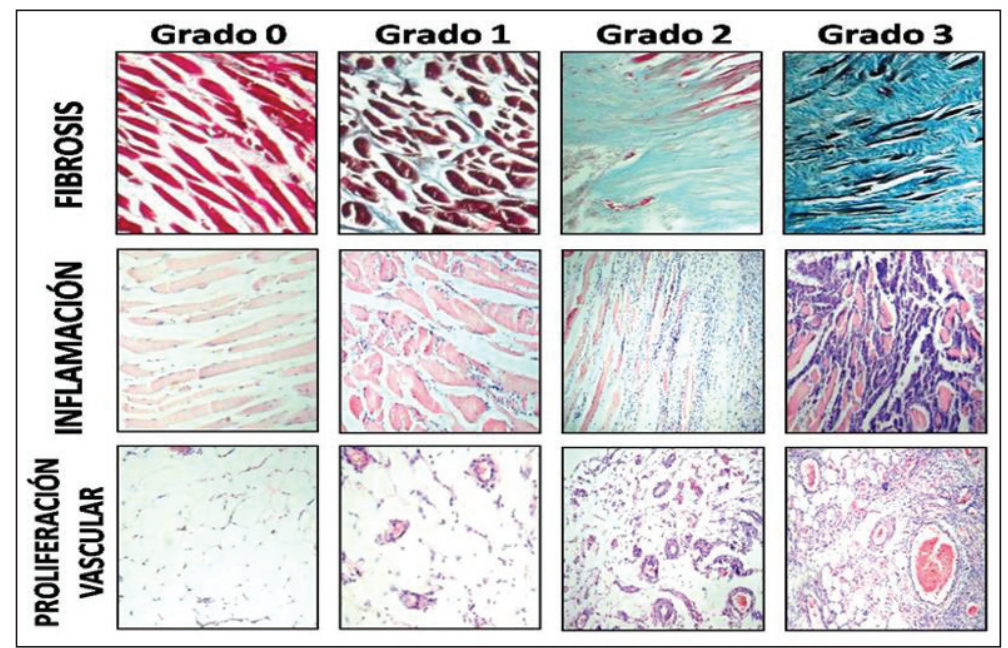

Figura 10. Cambios histológicos en adherencias peritoneales postoperatorias.

\section{Discusión}

Las AP, se consideran como una complicación mayor de la cirugía abdomino-pélvica, con una morbi-mortalidad importante, que ha originado esfuerzos de investigación múltiples, en la búsqueda de un tratamiento preventivo ideal. Varios agentes han sido estudiados, incluyendo el uso de antiinflamatorios, antioxidantes, anticoagulantes y fibrinolíticos ${ }^{21}$.

En años recientes, se han realizado múltiples investigaciones determinando el proceso fisiopatológico responsable de la formación de las AP, y se ha evidenciando que el sistema fibrinolítico desempeña un rol fundamental en el desarrollo de las AP. En especial, se ha estudiado la SP, una taquicinina, cuya acción sobre los NK-R1 está directamente relacionada con múltiples efectos adhesiogénicos ${ }^{15,22}$. Basándose en este conocimiento, en años recientes, se han realizado diversos estudios de prevención de AP mediante la utilización de NK-R1A ${ }^{23-28}$, evidenciándose resultados similares a los obtenidos en el presente estudio, una disminución significativa de la formación de AP al compararla con un grupo control sin tratamiento. Dichos estudios han determinado que este efecto preventivo, está relacionado con un aumento de la actividad fibrinolítica peritoneal, demostrado mediante un aumento de los niveles ARNm de tPA, así como de la actividad del tPA, y disminución de los niveles de PAI-1 en el fluido peritoneal ${ }^{23-28}$. Sin embargo, en ninguno de estos estudios, se evalúa la severidad de las AP, sólo el número de las mismas; en el presente estudio se demostró disminución tanto del número como la severidad de las AP. Además, se evalúan los cambios histopatológicos posteriores al uso de NK-R1A, tratamiento que demostró presentar menores grados de fibrosis, inflamación y proliferación vascular que el grupo control. El uso intraperitoneal de un NK-R1A demostró ser eficaz en la disminución de AP no sólo en órganos manipulados durante la cirugía, sino que adicionalmente disminuyó las AP en los órganos no manipulados durante el transoperatorio, cualidad que supone una ventaja frente a los materiales físicos o de barrera, cuyo efecto preventivo está limitado únicamente al sitio de aplicación, permitiendo la formación de AP distales al sitio de colocación ${ }^{29}$.

En conclusión, hasta que no exista un método preventivo de AP, totalmente efectivo, es fundamental la realización de estudios que permitan entender a cabalidad los eventos fisiopatológicos involucrados en la formación de AP, que sin duda conducirán al desarrollo de métodos preventivos eficaces. A este respecto, el presente estudio determinó que la administración intraperitoneal de un agente farmacológico, que actúa como antagonista de los NK-R1, constituye una opción promisoria, ya que se basa en el conocimiento fisiopatológico del sistema fibrinolítico peritoneal y tiene alta eficacia previendo la formación de AP. En vista de esto, es necesario mantener líneas de investigación que evalúen, tanto los mecanismos de acción como los posibles efectos adversos de esta droga en la prevención de AP.

\section{Referencias}

1. Vrijland WW, Jeekel J, van Geldorp HJ, Swank DJ, Bonjer HJ. Abdominal adhesions: intestinal obstruction, pain, and infertility. Surg Endosc 2003; 17: 1017-1022.

2. Sulaiman H, Dawson L, Laurent GJ, Belligan GJ, Herrik SE. Role of plasminogen activators in peritoneal adhesion formation. Biochem Soc Trans 2002; 30: 126-131. 
3. Vrijland WW, Tseng LN, Eijkman HJ, Hop WC, Jakimowicz JJ, Leguit P, et al. Fewer intraperitoneal adhesions with use of hyaluronic acid-carboxymethylcellulose membrane: a randomized clinical trial. Ann Surg 2002; 235: 193-199.

4. Dijkstra FR, Nieuwenhuijzen M, Reijnen MM, van Goor H. Recent clinical developments in pathophysiology, epidemiology, diagnosis and treatment of intraabdominal adhesions. Scand J Gastroenterol Suppl 2000; 232: 52-59.

5. Attard J, MacLean A. Adhesive small bowel obstruction: epidemiology, biology and prevention. Can J Surg 2007; 50: 291-300.

6. Menzies D, Ellis H. Intestinal obstruction from adhesions-how big is the problem? Ann R Coll Surg Engl 1990; 72: 60-63.

7. Johnston S, O'Malley K, McEntee G, Grace P, Smyth E, Bouchier-Hayes D. The need to retrieve the dropped stone during laparoscopic cholecystectomy. Am J Surg 1994; 167: 608-610.

8. Bryant T. Clinical lectures on intestinal obstruction. Med Tim Gaz 1: 363-365.

9. Soybel DI. Ileus and bowel obstruction. In: Greenfield LJ, editor. Surgery: scientific principles and practice. Philadelphia: Lippincott-Raven; 1997. p. 817e31.

10. Ergul E, Korukluoglu B. Peritoneal adhesions: Facing the enemy. Int J Surg 2008; 6: 253-260.

11. Becker JM, Dayton MT, Fazio VW, Beck DE, Stryker SJ, Wexner SD, et al. Prevention of postoperative abdominal adhesions by a sodium hyaluronate-based bioresorbable membrane: a prospective, randomized, double-blind multicenter study. J Am Coll Surg 1996; 183: 297-306.

12. Beck DE, Cohen Z, Fleshman JW, Kaufman HS, van Goor H, Wolff BG. A prospective, randomized, multicenter, controlled study of the safety of Seprafilm adhesion barrier in abdominopelvic surgery of the intestine. Dis Colon Rectum 2003; 46: 1310-1319.

13. Bahadir I, Oncel M, Kement M, Sahip Y. Intra-Abdominal use of taurolidine or heparin as alternative products to an antiadhesive barrier (Seprafilm) in adhesión prevention: An experimental study on mice. Dis Colon Rectum 2007; 50: 2209-2214.

14. Zeng Q, Yu Z, You J, Zhang Q. Efficacy and Safety of Seprafilm for Preventing Postoperative Abdominal Adhesion: Systematic Review and Meta-analysis. World J Surg 2007; 31: 2125-2131.

15. Cheong YC, Laird SM, Li TC, Shelton JB, Ledger WL, Cooke ID. Peritoneal healing and adhesion formation/ reformation. Hum Reprod Update 2001; 7: 556-566.

16. Reed K, Fruin A, Bishop-Bartolomei K, Gower A, Nicolaou M, Stucchi A, et al. Neurokinin-1 receptor and substance P Messenger RNA levels increase during intraabdominal adhesions formation. J Surg Res 2002;
108: $165-172$.

17. Gaertner W, Hagerman H, Felemovicius I, Bonsack M, Delaney J. Two experimental models for generating abdominal adhesions. J Sur Res 2008; 146: 241-245.

18. Oncel M, Remzi F, Connor J, Fazio V. Comparision of cecal abrasión and multiple-abrasion models generating intra-abdominal adhesions for animal studies. Tech Coloproctol 2005; 9: 29-33.

19. Mazuji MK, Calazmbaheti K, Pover B. Prevention of adhesions with polivinil pyrrolidone. Arch Surgery 1964; 89: 1011-1015.

20. Kaya U, Oktem M, Zeynelaglu H, Ozen O, Kuscu E. Impact of aromatase on adhesión formation in a rat model. Fertil Steril 2007; 87: 934-939.

21. Boland G, Weigel R. Formation and prevention of postoperative adhesions. J Surg Res 2006; 132: 3-12.

22. Sulaiman H, Gabella G, Davis MC, Mutsaers SE, Boulos P, Laurent GJ, et al. Presence and distribution of sensory nerve fibers in human peritoneal adhesions. Ann Surg 2001; 234: 256-261.

23. Reed K, Fruin A, Gower A, Stucchi A, Leeman S, Becker JA. Neurokinin 1 receptor antagonist decreases postoperative peritoneal adhesión formation and increase peritoneal fibrinolytic activity. PNAS 2004; 101 : 9115-9120.

24. Cohen PA, Aarons CB, Gower AC, Stucchi AF, Leeman $\mathrm{SE}$, Becker JM, et al. The effectiveness of a single intraperitoneal infusión of a neurokinin-1 receptor antagonist in reducing postoperative adhesión formation is time dependent. Surgery 2007; 141: 368-375.

25. Prushik SG, Aarons CB, Matteotti R, Reed KL, Gower $\mathrm{AC}$, Leeman SE, et al. A neurokinin 1 receptor antagonist decreases adhesión reformation after laparoscopic lysis of adhesions in a rat model of adhesion formation. Surg Endosc 2007; 21: 1790-1795.

26. Lim R, Morrill JM, Prushik SG, Reed KL, Gower AC, Leeman SE, et al. An FDA Approved Neurokinin-1 Receptor Antagonist is Effective in Reducing Intraabdominal Adhesions when Administered Intraperitoneally, But Not Orally. J Gastrointest Surg 2008; 12: 1754-1761.

27. Reed KL, Heydrick SJ, Aarons CB, Prushik S, Gower AC, Stucchi AF, et al. A neurokinin-1 receptor antagonist that reduces intra-abdominal adhesions decreases oxidative stress in the peritoneum. Am J Physiol Gastrointest Liver Physiol 2007; 293: 544-551.

28. Cohen PA, Gower AC, Stucchi AF, Leeman SE, Becker JM, Reed KL. A neurokinin-1 receptor antagonist that reduces intraabdominal adhesion formation increases peritoneal matrix metalloproteinase activity. Wound Repair Regen 2007; 15: 800-808.

29. Rizal L, Morrill J, Lynch R, Reed K, Gower A, Leeman $\mathrm{S}$, et al. Practical limitations of bioresorbable membranes in the prevention of intra-abdominal adhesions $\mathrm{J}$ Gastrointest Surg 2009; 13: 35-42. 